\title{
Alternative derivations for the fields inside a waveguide
}

\author{
Raghavendra G. Kulkarni ${ }^{1}$
}

\begin{abstract}
Generally, the longitudinal magnetic field of the transverse electric (TE) wave inside a waveguide is obtained by solving the corresponding Helmholtz wave equation, which further leads to the derivation of the remaining fields. In this paper, we provide an alternative way to obtain this longitudinal magnetic field by making use of one of the Maxwell's equations instead of directly relying on the Helmholtz wave equation. The longitudinal electric field of the transverse magnetic (TM) wave inside a waveguide can also be derived in a similar fashion. These derivations, which are different from those found in the introductory textbooks on microwave engineering, make the study of waveguides more interesting.
\end{abstract}

K e y w ords: Maxwell's equations, Helmholtz wave equation, waveguides, TE wave, TM wave

\section{Introduction}

The theory of the propagation of electromagnetic waves through transmission lines was developed by Oliver Heaviside in 1893; however he was of the opinion that two wires are necessary for guiding the electromagnetic waves, and ruled out the possibility of propagation of waves inside hollow tubes (without a center conductor). Later in 1897, Lord Rayleigh, who had interest in the study of various types of wave phenomena, presented a mathematical proof that the hollow tubes can support propagation of electromagnetic waves. But this proof was almost forgotten, and no significant follow up research surfaced for the next forty years. In 1936, George C. Southworth of Bell Telephone Laboratories and Wilmer L. Barrow of Massachusetts Institute of Technology, working independently and unaware of each other's work, presented simultaneously the theory of propagation of electromagnetic waves inside hollow waveguides, in a joint meeting of the American Physical Society (APS) and the Institute of Radio Engineers (IRE) [1].

Soon it was realized that waveguides are not just a means for the transmission of electromagnetic energy, but a wide range of microwave components, like antennas, filters, power dividers, directional couplers, etc, can be designed using these structures. For more details on the latest developments on various microwave components using waveguides, see the recent review paper [2]. The observation of the phenomenon of radiation of electromagnetic waves from the slots carved on the waveguides led to the development of various types of slot antennas; the slots can be either longitudinal or transverse to the direction of propagation [3-5]. Another microwave component that finds extensive use in terrestrial as well as space communication systems is the waveguide filter, which has high power-handling capability and it is rugged in construction. The waveguide filters can be made compact by designing them for the multimode and nonresonant mode operations $[2,6]$. Waveguide filters used in satellites need to be of light weight as well, hence they are often fabricated using special materials like Kelvar and graphite instead of the traditional material, aluminium [7].

The fields inside the waveguide structures are well dealt in several textbooks on microwave engineering, for example see [8-10]. This note presents a new approach to obtain the fields inside a rectangular waveguide, which is not generally dealt explicitly in the textbooks.

\section{A new approach to obtain waveguide fields}

It is well known that a waveguide, either a rectangular or a circular, supports transverse electric (TE) and transverse magnetic (TM) wave propagation, but not the transverse electromagnetic (TEM) wave propagation. This is due to the fact that the transverse electric and magnetic fields inside the waveguide are the functions of the longitudinal electric and magnetic fields. So when both the longitudinal fields are set to zero, which is an essential criteria for a TEM wave propagation, the transverse fields also vanish and consequently there is no wave propagation. Therefore, one of the longitudinal fields, either electric or magnetic, must exist for maintaining the transverse fields to ensure wave propagation inside the waveguide [8-10].

This note presents an alternative approach to determine the fields inside a waveguide, which is not normally dealt in the introductory textbooks on microwave engineering. In the first half of the note, the traditional approach to derive the fields inside the waveguide is given; and in the second half, the new approach is presented.

\footnotetext{
${ }^{1}$ Department of Electronics \& Communication Engineering, PES University, 100 Feet Ring Road, BSK III Stage, Bengaluru - 560085, India, raghavendrakulkarni@pes.edu; dr_rgkulkarni@yahoo.com
}

https://doi.org/10.2478/jee-2021-0018, Print (till 2015) ISSN 1335-3632, On-line ISSN 1339-309X (C) This is an open access article licensed under the Creative Commons Attribution-NonCommercial-NoDerivs License (http: //creativecommons.org/licenses/by-nc-nd/3.0/). 




Fig. 1. Rectangular waveguide

We begin our discussion by stating the four Maxwell's equations in the phasor form with the standard notations as shown below [8].

$$
\begin{gathered}
\nabla \times \mathbf{H}=j \omega \epsilon \mathbf{E}+\mathbf{J}, \quad \nabla \times \mathbf{E}=-j \omega \mu \mathbf{H}, \\
\nabla \cdot \mathbf{D}=\rho, \quad \nabla \cdot \mathbf{B}=0 .
\end{gathered}
$$

In the region inside a waveguide (which is source free) the current density $\mathbf{J}$ and the charge density $\rho$ are zero, so the Maxwell's equations given in (1) become,

$$
\begin{aligned}
\nabla \times \mathbf{H}=j \omega \epsilon \mathbf{E}, & \nabla \times \mathbf{E}=-j \omega \mu \mathbf{H}, \\
\nabla \cdot \mathbf{D}=0, \quad \nabla \cdot \mathbf{B} & =0 .
\end{aligned}
$$

Consider a rectangular waveguide as shown in Fig. 1. The wave propagation inside the waveguide is assumed to be in $+z$ direction, due to which all the fields will have $e^{-j \beta z}$ dependence, where $\beta$ is the phase constant of the propagating wave. For example, the variation of $x$ component of the electric field $\left(E_{x}\right)$ along the $z$ direction is given by $E_{x}=E_{x 0} e^{-j \beta z}$, where $E_{x 0}$ is the value of $E_{x}$ at $z=0$. Notice that the transverse fields inside the waveguide $\left(E_{x}, E_{y}, H_{x}\right.$, and $\left.H_{y}\right)$ can be expressed in terms of the longitudinal fields $\left(E_{z}\right.$ and $\left.H_{z}\right)$ by making use of the first two Maxwell's equations mentioned in (2):

$$
\begin{aligned}
& E_{x}=-\left(\frac{j \beta}{k_{c}^{2}}\right) \frac{\partial E_{z}}{\partial x}-\left(\frac{j \omega \mu}{k_{c}^{2}}\right) \frac{\partial H_{z}}{\partial y}, \\
& E_{y}=-\left(\frac{j \beta}{k_{c}^{2}}\right) \frac{\partial E_{z}}{\partial y}+\left(\frac{j \omega \mu}{k_{c}^{2}}\right) \frac{\partial H_{z}}{\partial x}, \\
& H_{x}=\left(\frac{j \omega \epsilon}{k_{c}^{2}}\right) \frac{\partial E_{z}}{\partial y}-\left(\frac{j \beta}{k_{c}^{2}}\right) \frac{\partial H_{z}}{\partial x}, \\
& H_{y}=-\left(\frac{j \omega \epsilon}{k_{c}^{2}}\right) \frac{\partial E_{z}}{\partial x}-\left(\frac{j \beta}{k_{c}^{2}}\right) \frac{\partial H_{z}}{\partial y},
\end{aligned}
$$

where $k_{c}^{2}=k^{2}-\beta^{2}$ and $k^{2}=\omega^{2} \mu \epsilon$. The quantities $k_{c}$ and $k$ are known as the cut-off wave number of the waveguide and the wave number of the medium filling the waveguide respectively. The medium inside the waveguide is assumed to be a perfect dielectric, and is characterized by its permeability $\mu$, permittivity $\epsilon$, and zero conductivity. For the propagation of TE waves, the criteria $E_{z}=0$ and $H_{z} \neq 0$ must be met; and for the propagation of TM waves, the criteria $H_{z}=0$ and $E_{z} \neq 0$ must be satisfied.

Let us first consider TE wave propagation inside the waveguide. Substituting $E_{z}=0$ in (3) to (6), we obtain the following expressions for the transverse fields in terms of $H_{z}$.

$$
\begin{aligned}
& E_{x}=-\left(\frac{j \omega \mu}{k_{c}^{2}}\right) \frac{\partial H_{z}}{\partial y}, \quad E_{y}=\left(\frac{j \omega \mu}{k_{c}^{2}}\right) \frac{\partial H_{z}}{\partial x} \\
& H_{x}=-\left(\frac{j \beta}{k_{c}^{2}}\right) \frac{\partial H_{z}}{\partial x}, \quad H_{y}=-\left(\frac{j \beta}{k_{c}^{2}}\right) \frac{\partial H_{z}}{\partial y} .
\end{aligned}
$$

Notice that the field $H_{z}$ has to be determined first in order to determine the other fields. For this purpose, the traditional approach followed is to consider the Helmholtz equation in $H_{z}$ as shown below,

$$
\left(\frac{\partial^{2}}{\partial x^{2}}+\frac{\partial^{2}}{\partial y^{2}}+\frac{\partial^{2}}{\partial z^{2}}+k^{2}\right) H_{z}=0
$$

Since $H_{z}=H_{z 0} e^{-j \beta z}$, where $H_{z 0}$ is $H_{z}$ at $z=0$, the Helmholtz equation (8) becomes,

$$
\left(\frac{\partial^{2}}{\partial x^{2}}+\frac{\partial^{2}}{\partial y^{2}}+k_{c}^{2}\right) H_{z 0}=0
$$

where $k_{c}^{2}=k^{2}-\beta^{2}$. Solving (9) and applying the boundary conditions, the longitudinal field $H_{z}$ is determined as,

$$
H_{z}=A^{\prime} \cos \frac{m \pi x}{a} \cos \frac{n \pi y}{b} e^{-j \beta z},
$$

where $A^{\prime}$ is some arbitrary constant, $m$ and $n$ take integer values depending on the mode of operation. The quantities, $a$ and $b$ are the width and the height of the rectangular waveguide, see Fig. 1 . Using $H_{z}$ we determine the remaining fields from the expressions mentioned in (7). For the derivation of (10) from (9), one may refer the textbooks (see [8-10]); so it is not discussed here.

The intent of this note is to obtain expression (9) by an alternative approach without relying on the Helmholtz equation (8). For this purpose we make use of the Maxwell's equation, $\nabla \cdot \mathbf{B}=0$, to determine $H_{z}$. Observe that $\nabla \cdot \mathbf{B}=0$ implies $\nabla \cdot \mathbf{H}=0$, which when expanded yields,

$$
\frac{\partial H_{x}}{\partial x}+\frac{\partial H_{y}}{\partial y}+\frac{\partial H_{z}}{\partial z}=0
$$

We know that $H_{z}=H_{z 0} e^{-j \beta z}$, so, $\partial H_{z} / \partial z=-j \beta H_{z}$. The expressions for $H_{x}$ and $H_{y}$ are given in (7). Differentiating $H_{x}$ with respect to $x, H_{y}$ with respect to $y$, and using them in (11) yields,

$$
-\left(\frac{j \beta}{k_{c}^{2}}\right) \frac{\partial^{2} H_{z}}{\partial x^{2}}-\left(\frac{j \beta}{k_{c}^{2}}\right) \frac{\partial^{2} H_{z}}{\partial y^{2}}-j \beta H_{z}=0,
$$

which after some manipulations results in,

$$
\left(\frac{\partial^{2}}{\partial x^{2}}+\frac{\partial^{2}}{\partial y^{2}}+k_{c}^{2}\right) H_{z 0}=0
$$


Notice that the above expression is identical to (9) and this approach is generally not found in the textbooks.

For the case of TM waves, we substitute $H_{z}=0$ in the expressions, (3) to (6), to obtain the transverse fields in terms of $E_{z}$, as follows:

$$
\begin{aligned}
& E_{x}=-\left(\frac{j \beta}{k_{c}^{2}}\right) \frac{\partial E_{z}}{\partial x}, \quad E_{y}=-\left(\frac{j \beta}{k_{c}^{2}}\right) \frac{\partial E_{z}}{\partial y}, \\
& H_{x}=\left(\frac{j \omega \epsilon}{k_{c}^{2}}\right) \frac{\partial E_{z}}{\partial y}, \quad H_{y}=-\left(\frac{j \omega \epsilon}{k_{c}^{2}}\right) \frac{\partial E_{z}}{\partial x} .
\end{aligned}
$$

To determine the field $E_{z}$, the textbooks make use of the Helmholtz equation in $E_{z}$ and proceed to solve it. Instead, here we use the Maxwell's equation, $\nabla \cdot \mathbf{D}=0$, in the source free region [see (2)]. This implies $\nabla \cdot \mathbf{E}=0$, which when expanded yields,

$$
\frac{\partial E_{x}}{\partial x}+\frac{\partial E_{y}}{\partial y}+\frac{\partial E_{z}}{\partial z}=0 .
$$

Notice that $\partial E_{z} / \partial z=-j \beta E_{z}$. The expressions for $E_{x}$ and $E_{y}$ are given in (12); differentiating $E_{x}$ with respect to $x, E_{y}$ with respect to $y$, and using them in (13) yields,

$$
-\left(\frac{j \beta}{k_{c}^{2}}\right) \frac{\partial^{2} E_{z}}{\partial x^{2}}-\left(\frac{j \beta}{k_{c}^{2}}\right) \frac{\partial^{2} E_{z}}{\partial y^{2}}-j \beta E_{z}=0,
$$

which after some manipulations leads to

$$
\left(\frac{\partial^{2}}{\partial x^{2}}+\frac{\partial^{2}}{\partial y^{2}}+k_{c}^{2}\right) E_{z 0}=0
$$

The field $E_{z}$ is obtained after solving (14) and making use of the boundary conditions at the waveguide walls,

$$
E_{z}=B^{\prime} \sin \frac{m \pi x}{a} \sin \frac{n \pi y}{b} e^{-j \beta z},
$$

where $B^{\prime}$ is some arbitrary constant, $m$ and $n$ take integer values depending on the mode of operation. Using (15) the remaining fields are determined from (12). The derivation of (15) using (14) is available in the textbooks [8-10]; readers are advised to refer them.

In summary, this note provides an alternative approach to obtain the fields inside a waveguide; while the textbooks make use of the Helmholtz equation, here we use the Maxwell's equations, $\nabla \cdot \mathbf{D}=0$, and $\nabla \cdot \mathbf{B}=0$, to derive these fields.

\section{Acknowledgments}

The author thanks the management of PES University for supporting this work.

\section{REFERENCES}

[1] K. Packard, "The Origin of Waveguides: A Case of Multiple Rediscovery", IEEE Transactions on Microwave Theory and Techniques, vol. 32, no. 9, pp. 961-969, 1984. Available: 10.1109/tmtt.1984.1132809.

[2] S. Wong, J. Lin, Y. Yang, Z. Guo, L. Zhu, and Q. Chu, "Waveguide Components Based on Multiple-Mode Resonators: Advances in Microwave Multiple-Mode Waveguide Components, Including Multiplexers, Three-State Diplexers, Crossovers, and Balanced/Unbalanced Elements", IEEE Microwave Magazine, vol. 22, no. 2, pp. 33-45, 2021. Available: $10.1109 / \mathrm{mmm} .2020 .3035863$.

[3] W. Watson, "Resonant Slots", Journal of the Institution of Electrical Engineers - Part IIIA: Radiolocation, vol. 93, no. 4, pp. 747-777, 1946. Available: 10.1049/ji-3a-1.1946.0167.

[4] R. Elliott and W. O'loughlin, "The Design of Slot Arrays Including Internal Mutual Coupling", IEEE Transactions on Antennas and Propagation, vol. 34, no. 9, pp. 1149-1154, 1986, Available: 10.1109/tap.1986.1143947.

[5] L. Josefsson, "A Waveguide Transverse Slot for Array Applications", IEEE Transactions on Antennas and Propagation, vol. 41, no. 7, pp. 845-850, 1993. Available: 10.1109/8.237613.

[6] S. Bastioli, "Nonresonating Mode Waveguide Filters", IEEE Microwave Magazine, vol. 12, no. 6, Oct 2011, pp. 77-86, 2011. Available:10.1109/mmm.2011.942009.

[7] V. Boria and B. Gimeno, "Waveguide Filters for Satellites", IEEE Microwave Magazine, vol. 8, no. 5, pp. 60-70, 2007. Available: $10.1109 / \mathrm{mmm} .2007 .903649$.

[8] E. Jordan and K. Balmain, Electromagnetic Waves and Radiating Systems, 2nd ed. New Delhi: Prentice-Hall of India, 2009.

[9] D. Pozar, Microwave Engineering, 4th ed. New Jersey: John Wiley \& Sons Inc., 2012.

[10] R. Collin, Foundations for Microwave Engineering, 2nd ed. New Delhi: Wiley (India), 2005.

Received 5 March 2021

Raghavendra G. Kulkarni obtained his BE degree in Electronics and Communication Engineering from Karnatak University, his MTech degree from IIT Madras, and his PhD degree from IIT Kharagpur. He joined Hybrid Microcircuits division of Bharat Electronics Ltd., Bengaluru in 1982, and was involved in the design of Surface-Acoustic-Wave (SAW) filters. He obtained two patents on SAW filter design and has authored about seventy papers in the field of SAW filters, electromagnetics, signal processing, and mathematics. He is the inventor of several window functions for signal processing applications. He has conducted SAW filter design workshops at URSC / ISRO and CRL / BEL in Bengaluru. He has given invited talks at IISC Bengaluru, LRDE Bengaluru, TIFR Mumbai, VSSC Trivandrum, IGCAR Kalpakkam, etc. In 2014, He joined PES University, Bengaluru, as Distinguished Professor in the Department of Electronics \& Communication Engineering and is a senior member of IEEE, life member of Ramanujan Mathematical Society, and Indian Mathematical Society. 